\title{
SYNCHROTRON AND COMPTON COMPONENTS AND THEIR VARIABILITY IN BL LAC OBJECTS
}

\author{
P.Giommi ${ }^{1}$, G. Ghisellini ${ }^{2}$, P. Padovani ${ }^{3,4}$, and G. Tagliaferri ${ }^{2}$ \\ 1) BeppoSAX Science Data Center, Via Corcolle 19, 00131 Rome, Italy \\ 2) Osservatorio Astronomico di Brera, Via Bianchi 46, 23807 Merate, Italy \\ 3) Space Telescope Science Institute, San Martin Drive, Baltimore, MD 21218 U.S.A. \\ 4) Affiliated to the Astrophysics Division, Space Science Department, European Space \\ Agency
}

\section{INTRODUCTION}

BL Lacertae objects are extreme extragalactic sources characterized by the emission of strong and rapidly variable nonthermal radiation over the entire electromagnetic spectrum. Synchrotron emission followed by inverse Compton scattering in a relativistic beaming scenario is generally thought to be the mechanism powering these objects (e.g. Kollgaard 1994., Urry \& Padovani 1995). BL Lacs can be divided into different subclasses depending on their Spectral Energy Distribution (SED), namely LBL for objects with the synchrotron emission peaking at $\nu_{\text {peak }} \approx 10^{13-14} \mathrm{~Hz}$, intermediate objects $\left(\nu_{\text {peak }} \approx 10^{15-16} \mathrm{~Hz}\right)$ and HBL or high energy peaked BL Lacs with $\nu_{\text {peak }} \approx 10^{17-18} \mathrm{~Hz}$ (Padovani \& Giommi 1995). The wide X-ray band pass of the BeppoSAX satellite (Boella et al. 1997) is well suited for the detailed spectral study of all types of BL Lacs. In fact, direct measurements of the Compton part of the spectrum have been obtained for a number of LBLs (e.g. Padovani et al. 1999), and the very variable tail of the Synchrotron component has been studied in several HBLs (e.g. Pian et al. 1998, Wolter et al. 1998, Giommi, Padovani \& Perlman 1999, Chiappetti et al. 1999). In the case of the two intermediate BL Lacs S5 0716+714 and ON 231 BeppoSAX for the first time was able to detect both spectral components within a single instrument (Giommi et al. 1999, Tagliaferri et al. 1999).

The BeppoSAX archive at the Science Data Center (SDC, Giommi \& Fiore 1998) presently includes over 100 observations of 56 distinct BL Lacs, about half of which are already publicly available. We have started a project to construct the SED of a large number of all types of BL Lacs by combining a) public BeppoSAX data (0.1-200 keV); b) simultaneous optical and radio data when these are available from monitoring campaigns, or from the University of Michigan Radio Astronomy Observatory (UMRAO) on-line data base (Aller et al. 1999); and c) non-simultaneous photometric data form NED. Here we present the first results of this project. 

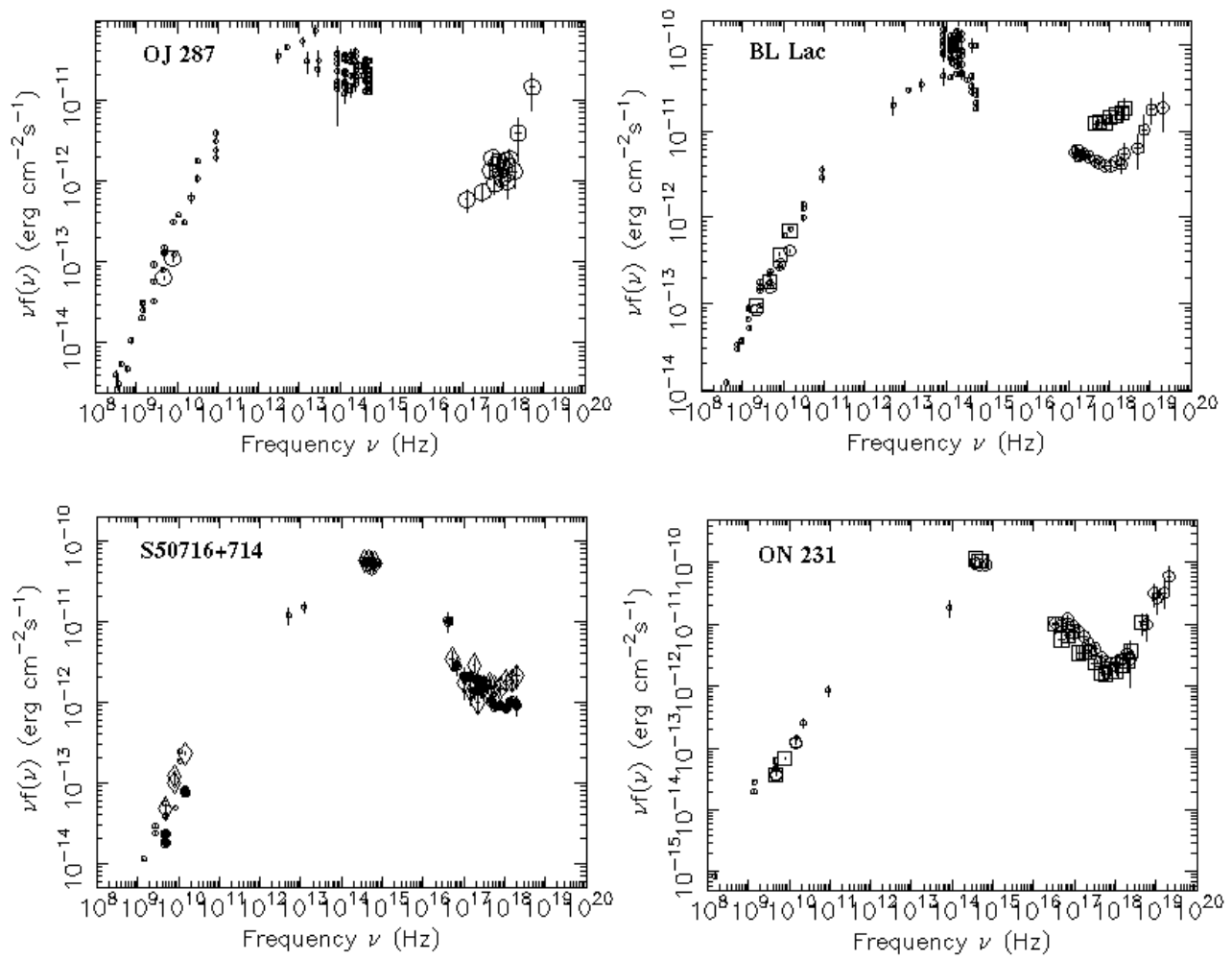

FIGURE 1. Spectral energy distribution of four LBL and intermediate BL Lacs.

\section{SPECTRAL ENERGY DISTRIBUTIONS AND VARIABILITY}

The SEDs that we have assembled are shown in figure 1 for LBLs and intermediate objects, and in figure 2 for HBL BL Lacs. The X-ray part of the plots have been constructed using data from the LECS, MECS and PDS instruments of the BeppoSAX satellite. The cleaned and calibrated data files have been taken from the SDC on-line archive and have been analyzed using the XSPEC package. Unfolded spectral data have been corrected for low energy absorption assuming $N_{H}$ equal to the Galactic value. Nearly simultaneous data are plotted with the same symbols used for the X-ray data. Optical monitoring observations are available for S5 0716+714 (Giommi et al. 1999), and ON 231 (Tagliaferri et al. 1999). Nearly simultaneous radio data from the UMRAO database are available for several objects. All other (non-simultaneous) data are plotted as small open circles and are from the

photometric data points provided by NED. Strong variability at several frequencies is apparent from Figures 1 and 2. In particular quite spectacular spectral changes are concentrated at or just after the synchrotron peak. BeppoSAX observations of 

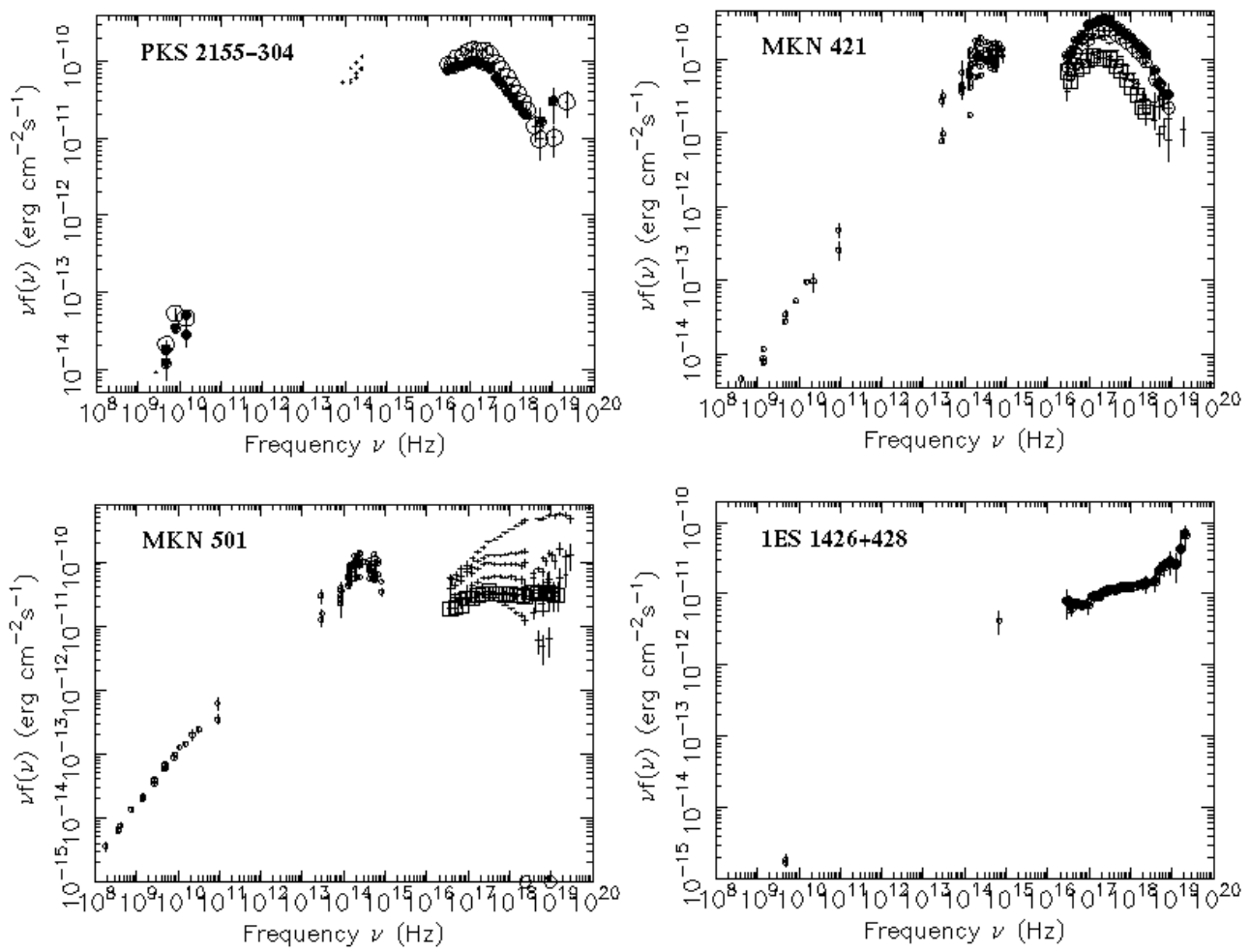

FIGURE 2. Spectral energy distribution of HBL BL Lacs.

intermediate BL Lacs clearly show that the soft X-ray synchrotron radiation vary in a different way compared to the harder Compton components (Giommi et al. 1999, Tagliaferri et al. 1999). The SED shown here indicate that the variability of the Compton component may be correlated with radio flux and not with the optical and soft X-ray synchrotron emission (see figure 1).

\section{ULTRA HIGH ENERGY SYNCHROTRON PEAKED BL LACS (UHBLS) ?}

Figures 1 and 2 clearly show that the peak frequency of the synchrotron emission ranges from around $10^{13} \mathrm{~Hz}$ for OJ 287 to well above $10^{19} \mathrm{~Hz}$ for $1 \mathrm{ES} 1426+428$. Ghisellini (1999) argued that this trend could continue to much higher energies. We have thus been searching for BL Lacs with Ultra High synchrotron peak energy (UHBLs). We have selected candidates UHBLs from the sample of extreme BL Lacs of the "Sedentary Multifrequency Survey" (Giommi, Menna \& Padovani 1999) by looking for objects within the error circle of unidentified sources in the third EGRET catalog. One such object is 1RXS J23511.1-14033; its finding chart is shown in figure 3 (left). The SED of 1RXS J23511.1-14033, on the right part of figure 3, indicates 

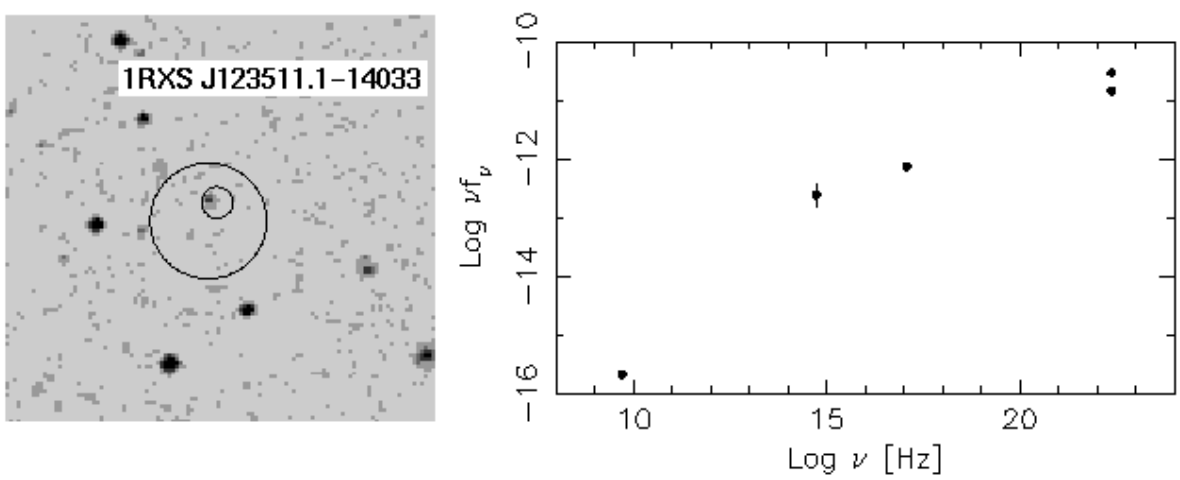

FIGURE 3. Left: the ROSAT and NVSS error circles showing the candidate UHBL 1RXS J123511.1-14033. Right: the SED of 1RXS J123511.1-14033 if this BL Lac is the correct counterpart of the EGRET source 2EGJ1233-1407

that the synchrotron emission could reach the gamma ray band. A first BeppoSAX pointing of this object unfortunately gave inconclusive results since the observation had to be split into three short exposures and the spectrum appears to be variable. Details will be published elsewhere. A second UHBL candidate will be observed by BeppoSAX in a few months. If these observations will confirm the hypothesis that UHBLs exist, this type of sources could be the long sought counterpart of many of the still unidentified high galactic latitude EGRET sources.

\section{REFERENCES}

Aller M.F, Aller H.D., Huges P.A., \& Latimer G.E., 1999 ApJ 512, 601

Boella G. et al. 1997 A\&AS, 122, 299

Chiappetti,L., et al. 1999, ApJ 521, 552

Ghisellini, G., 1999, Proc 3rd Integral Workshop, Taormina, astro-ph/9812419

Giommi P.,\& Fiore F. 1998, in Proc. 5th Workshop on Data Analysis in Astronomy, World Scientific, Singapore, p. 93

Giommi, P., Padovani, P. \& Perlman, E. 1999, MNRAS in press, astro-ph/9907377

Giommi, P. et al. 1999, A\&A, in press, astro-ph/9909241

Giommi, P., Menna, M.T., \& Padovani, P. 1999, MNRAS in press, astro-ph/9907014

Kollgaard R.I., 1994 Vistas in Astronomy, 38, 29

Padovani, P. \& Giommi, P. 1995, ApJ, 444, 567

Padovani, P. et al. 1999, in preparation

Pian, E. et al. 1998, APJ L,492, L17

Tagliaferri, G., et al. 1999, A\&A, submitted

Urry, C.M., \& Padovani, P., 1995, PASP, 107, 803

Wolter, A. et al. 1998 A\&A 335, 899 